\title{
Comparison of endoscopic ultrasound-guided fine-needle aspiration by capillary action, suction, and no suction methods: a randomized blinded study
}

\section{(ㄷ)(1) $\odot(9$}

\author{
Authors \\ Bhasin', Haimanti Sarin², Mridula Guleria², Randhir Sud ${ }^{1}$ \\ Institutions \\ 1 Institute of Digestive and Hepatobiliary Sciences, \\ Medanta the Medicity, Gurugram, India \\ 2 Department of Cytopathology, Medanta the Medicity, \\ Gurugram, India
}

Rinkesh K. Bansal' ${ }^{1}$, Narendra S. Choudhary ${ }^{1}$, Rajesh Puri ${ }^{1}$, Saurabh K. Patle ${ }^{1}$, Suraj Bhagat ${ }^{1}$, Mukesh Nasa ${ }^{1}$, Amit

submitted 17.3.2017

accepted after revision 23.6.2017

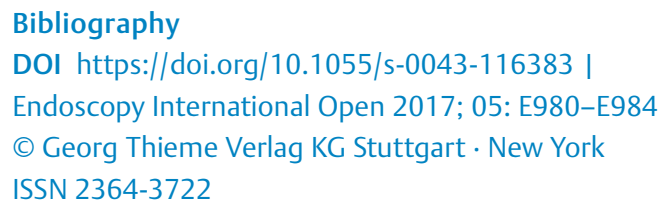

Corresponding author

Rajesh Puri, MD, Institute of Digestive and Hepatobiliary Sciences, Medanta The Medicity, Sector 38, Gurugram, Delhi NCR, India, PIN 122001

Fax: +91-012448-34111

purirajesh70@gmail.com

\section{ABSTRACT}

Background and study aim Different types of endoscopic ultrasound (EUS)-guided fine-needle aspiration (FNA) techniques are used in clinical practice; the best method in terms of outcome has not been determined.
The aim of the study was to compare the diagnostic adequacy of aspirated material, and the cytopathological and EUS morphological features between capillary action, suction, and no-suction FNA methods.

Patients and methods This was a prospective, singleblinded, randomized study conducted at a tertiary care hospital. Patients were randomized to the three groups: capillary action, suction, and no suction. A total of 300 patients were included, with 100 patients in each arm.

Results A total of 300 patients (195 males) underwent EUS-FNA of 235 lymph nodes and 65 pancreatic masses (distribution not statistically different between the groups). The mean age was $52 \pm 14$ years. A 22 gauge needle was used in the majority ( $93 \%$ ) of procedures. There was no statistical difference between the three groups regarding lymph node size at the largest axis and ratio, type of needle, echo features, echogenicity, calcification, necrosis, shape, borders (lymph nodes), number of passes, and cellularity. Diagnostic adequacy of the specimen was $91 \%$, $91 \%$, and $94 \%$ in the capillary, suction, and no suction groups, respectively $(P=0.67)$. Significantly more slides and blood clots were generated by the suction method compared with the other methods.

Conclusion The capillary action, suction, and no suction methods of EUS-FNA are similar in terms of diagnostic adequacy of the specimen. The suction method has the disadvantages of causing more bleeding and generating more slides.

\section{Introduction}

Endoscopic ultrasound-guided fine-needle aspiration (EUSFNA) is a high yielding and safe diagnostic modality to obtain a tissue diagnosis for various indications [1, 2]. Both lymph nodes and solid organs in the vicinity of the gastrointestinal tract can be approached with EUS-FNA. The sensitivity of EUS-FNA is more than $85 \%$, and is higher for nodes than for submucosal lesions [3].

During EUS-FNA, a needle is inserted into the area of interest and a sample is aspirated by various methods. Methods such as suction, no suction, and capillary action have been reported in the literature but without concluding which of the methods is best. The use of the stylet and suction have not been properly assessed, but it is clear that many operators continue to use the stylet despite extensive data proving its lack of value [4-8], and many continue to use suction despite conflicting data [912].

We conducted this study to compare the various methods of EUS-FNA - suction, no-suction, and capillary action. 


\section{Patients and methods}

\section{Inclusion criteria and study design}

The study was conducted from January 2015 to December 2015 at a tertiary care center in North India (Delhi NCR). All consecutive patients aged 18 years or more who underwent EUS-FNA of lymph nodes and/or solid mass were included in the analysis. Informed consent was obtained from all patients. Exclusion criteria included age less than 18 years, pregnancy, submucosal lesions, coagulopathy (international normalized ratio $>1.5$, platelets $<50000 / \mathrm{mm}^{3}$ ), and failure to provide informed consent for the procedure.

The study was conducted prospectively after clearance from the institute's review board/ethical committee. The study was single-blinded and randomized. The cytopathologists were blinded to the method of FNA (suction, no suction, or capillary action). Computer-generated randomization was done.

\section{EUS-FNA procedure}

All procedures were carried out with the patient under conscious sedation with midazolam, which was administered by an anesthesiologist. EUS-FNA was performed using the lineararray GF-UCT140 echoendoscope (Olympus, Tokyo, Japan) by experienced endosonographers (R.P., N.S.C.). EUS features such as echogenicity, calcification and necrosis, and shape and border of lymph nodes were recorded. For echogenicity, hypoor hyper- or mixed echogenicity was recorded. The shape of the lymph node was either round or oval, and borders were either ill-defined or sharply defined.

The FNA needle with stylet was introduced through the working channel. Doppler was used to avoid any vascular structures in the needle path. The stylet was withdrawn slightly before puncture. A 22-gauge needle (EchoTip Ultra HD; Cook Endoscopy, Winston-Salem, North Carolina, USA) was used in the majority of the patients; 19 and 25 gauge needles were used in the remaining patients.

The method of FNA - capillary action, suction or no suction - was selected by computer-based randomization and defined as follows: suction - after removing the stylet, a $10 \mathrm{~mL}$ suction syringe, loaded with maximal suction, was attached in a "locked" position to the needle and suction was applied after the lesion was punctured; no suction - suction was not applied after removing the stylet, before performing EUS-FNA; capillary action - suction was not applied as the stylet was removed gradually after the lesion was punctured.

Each pass consisted of 15-20 to-and-fro movements of the needle within the lesions, using the fanning technique whenever possible. A separate needle was used for different lesions if needed. Each of the lesions in the study were sampled using one of the three methods, following computer-generated randomization, with the same needle.

\section{Cytopathology}

After aspiration, the material inside the needle was pushed onto slides, little by little, with the help of the stylet. One or two slides from each pass were immediately fixed in absolute alcohol and the remaining slides were air-dried. The slides were stained with Papanicolaou, Giemsa stain, and Ziehl-Neelsen stain (when required) for the detection of acid-fast bacilli. No on-site cytopathologist facility was available.

The cytopathological specimens were graded on a previously validated scale for cellularity, blood contamination, and adequacy. Cellularity was assessed using a 3 -point scale $(0=$ no cells; 1 = sparsely cellular; 2 = moderately cellular). Blood contamination was graded on a 3 -point scale $(0=$ no blood; 1 = contaminated with red blood cells; 2 =blood clots present). Specimen adequacy was graded on a 2 -point scale $(0=$ inadequate aspirate for the cytopathologist to make a diagnosis; 1 =adequate aspirate). A final diagnosis was achieved if the specimen was positive for malignancy or granuloma or reactive.

A lymph node was considered reactive when FNA showed lymphoid cells in different stages of activation in the presence of adequate cellularity and absence of granulomas, necrosis, or malignant cells.

All study specimens were reviewed and graded by an experienced cytopathologist, who was blinded to the FNA method used but was provided with demographic information and clinical history of the patient.

\section{Data collection}

The following data were recorded for each patient and compared between three groups: age, sex, indication for procedure, site of lymph node or solid mass, echo features of lymph node, size at long axis and ratio, type of needle and number of needle passes, number of slides, result of FNA, adequacy of specimen for diagnosis, cellularity, blood contamination, and any procedural complications. In the case of multiple lymph nodes, the largest node or the node with sharply demarcated borders or hypoechoic in nature was preferred for FNA.

\section{Study end points}

The primary end point of the study was the adequacy of the aspirated material. Secondary end points were cytopathological diagnosis and contamination of the specimen with blood.

\section{Statistical methods}

To achieve a diagnostic yield of $94 \%$, a sample of 100 was adequate with $95 \%$ confidence level and $+/-0.05$ precision; thus 100 patients were included in each group. The data are presented here as number, percentage, mean (SD), and median with interquartile range (IQR). The three groups were compared using analysis of variance test (parametric data), Kruskal-Wallis test (nonparametric data) or Fisher's exact test (categorical data). A two-tailed $P$ value of $<0.05$ was considered to be significant. All statistical analyses was performed using SPSS, version 19 (IBM Corp. Armonk, New York, USA).

\section{Results}

A total of 337 patients were enrolled in the study after providing consent. A total of 37 patients were excluded owing to no informed consent $(n=16)$ and decompensated cirrhosis with 


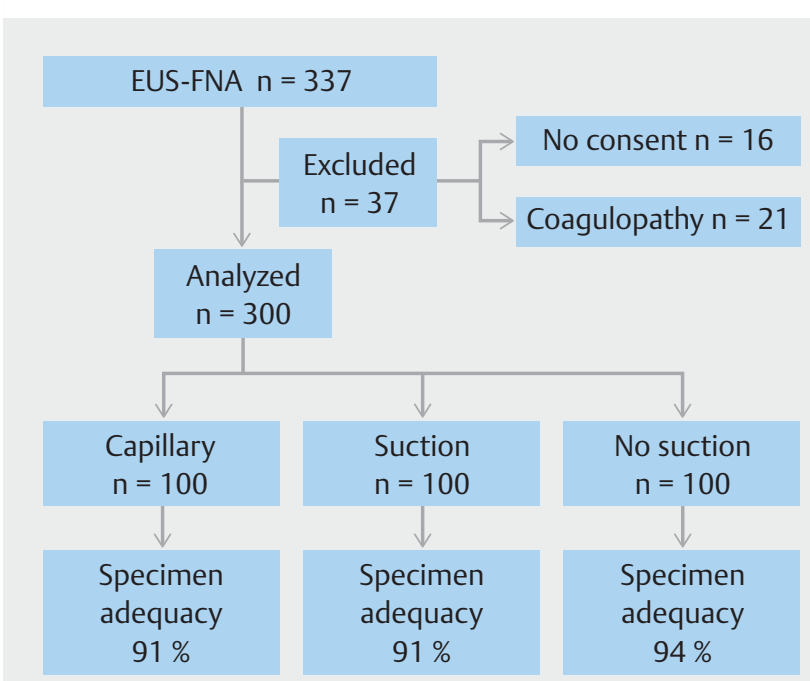

- Fig. 1 Flow diagram showing study design. EUS-FNA, endoscopic ultrasound-guided fine-needle aspiration.

coagulopathy $(n=21)$; the remaining 300 patients were included in the final analysis ( $\bullet$ Fig. 1 ).

The mean patient age was $52 \pm 14$ years and $65 \%$ were males $(\triangleright$ Table 1). FNA samples were taken from lymph nodes in 235 patients and from pancreatic masses in 65 patients. The distribution of samples was not statistically different among the three groups ( $\triangleright$ Table 1 ). A 22 gauge needle was used in the majority $(93 \%)$ of the patients. All three groups had a similar number of needle passes (median 2 in each group; not significant).

Diagnostic adequacy of the specimen was achieved in $92 \%$ of patients ( $91 \%, 91 \%$, and $94 \%$ of patients in the capillary, suction, and no suction groups, respectively; $P=0.67$ ). There was no statistically significant difference between the groups on subgroup analysis of diagnostic adequacy according to site (i.e. lymph node and pancreas) ( $\bullet$ Table 2 ).

A positive cytological diagnosis of malignancy was achieved in 97 patients, 112 patients had either granuloma or were positive for acid-fast bacilli, and 67 patients had reactive lymph node changes ( $\vee$ Table 1$)$. One patient had both granuloma and malignancy (granuloma in the lymph node and malignancy in the pancreas). In 24 patients, aspirates were labeled as suboptimal (20 lymph nodes, 4 pancreatic masses). Suboptimal, reactive, granulomatous, and malignancy were present in 9 , 24,35 , and 32 patients in the capillary group, in $9,17,41$, and 33 patients in the suction group, and in 6, 26, 36, and 32 patients in the no suction group, respectively; distribution was not statistically different between the three groups $(P=0.77)$ $(\triangleright$ Table 1 ).

There was no significant difference between the capillary, suction, and no suction groups regarding lymph node size at long axis (median $20 \mathrm{~mm}$ [IQR 12-30], $16 \mathrm{~mm}$ [14-24], and $19 \mathrm{~mm}$ [12-24], respectively; $P=0.15]$ and ratio (median 1.52 [IQR 1.33-2.00], 1.55 [1.40-2.00], and 1.50 [1.23-2.00], respectively; $P=0.25$ ] ( $\vee$ Table 1 ). There were no significant dif- ferences between the three groups in term of echogenicity, echo structure, calcification, necrosis, and shape and border of lymph nodes ( $\bullet$ Table 1 ).

In terms of cellularity, 4 patients had a score of 0,53 patients had a score of 1 , and 243 patients had a score of 2. Cellularity scores were 0,19 , and 81 in the capillary group, 2,14 , and 84 in the suction group, and 2, 20, and 78 in the no suction group, respectively $(P=0.49)(\vee$ Table 3 ).

The median (IQR) numbers of slides used were: $11(9-12)$ in the capillary group, $12(11-15)$ in the suction group, and 10 $(7-12)$ in the no suction group $(P<0.001)$. The suction group required significantly more slides.

In terms of blood contamination, there was no blood (score 0 ) in the specimens from 71 patients, contamination with red blood cells (score 1 ) in 174 patients, and clots (score 2) in 55 patients. Blood contamination scores were 28,63 , and 9 in the capillary group, 11, 59, and 30 in the suction group, and 32, 52, and 16 in the no suction group, respectively. Significantly more blood clots were observed in the suction group $(P<0.001)$ $(\triangleright$ Table 3$)$.

There were no FNA-related adverse events in any of the study groups.

\section{Discussion}

Martin and Ellis first presented their results of a tumor diagnosis by needle aspiration in 1930, by using an 18 gauge needle mounted onto a $20 \mathrm{~mL}$ syringe [13]. A similar technique was applied for EUS-FNA along with some modifications, including changes in needle design and use of variable amounts of suction, including capillary action.

Although EUS and EUS-guided FNA has become the modality of choice for examining lesions in the mediastinum and abdomen, and for obtaining tissue in the same sitting, sensitivity is highly variable and depends on many factors including the experience of the endosonographer. Whether the method of FNA has any impact on sensitivity and cellularity is largely unknown. Different types of EUS-FNA technique are used in clinical practice, but the best method in terms of outcome has not been determined.

In the current study, the suction group required significantly more slides and had more blood clots compared with the no suction and capillary action methods. The suction method applies a lot of pressure, which causes more tissue damage as well as bleeding, leading to an increase in the number of slides used. Suction improves cellularity and the quality of aspirate, but at the cost of increased bleeding and contamination [14]. Wallace et al. revealed that capillary action may improve specimen quality by reducing the amount of blood in the aspirated material [9]. The greater the number of slides generated by the acquisition process, the more time required by the pathologist to process and examine the specimens.

There was no significant difference between the three groups regarding lymph node size at long axis and ratio. There was no statistically significant difference between the three groups regarding type of needle used, echo features, adequacy of the specimen, and number of needle passes. 
- Table 1 Comparison of baseline characteristics and endoscopic ultrasound morphological features between the three methods of fine-needle aspiration.

\begin{tabular}{|c|c|c|c|c|}
\hline & Capillary & Suction & No suction & $P$ value \\
\hline Age, mean $\pm S D$, years & $52 \pm 14$ & $49 \pm 14$ & $53 \pm 15$ & 0.14 \\
\hline Sex, Male/Female, n & $65 / 35$ & $64 / 36$ & $66 / 34$ & 0.24 \\
\hline Site, Lymph node/Pancreas, n & $82 / 18$ & $82 / 18$ & $71 / 29$ & 0.08 \\
\hline Diagnostic adequacy, \% & 91 & 91 & 94 & 0.67 \\
\hline Diagnosis, Suboptimal/Reactive/Granulomatous/Malignancy) & $9 / 24 / 35 / 32$ & $9 / 17 / 41 / 33$ & $6 / 26 / 36 / 32$ & 0.77 \\
\hline Lymph node size at largest axis, median (IQR), mm & $20(12-30)$ & $16(14-24)$ & $19(12-24)$ & 0.15 \\
\hline Ratio, median (IQR) & $1.52(1.33-2.00)$ & $1.55(1.40-2.00)$ & $1.50(1.23-2.00)$ & 0.25 \\
\hline Echogenicity, Hypo/Hyper/Mixed, n & $60 / 16 / 24$ & $55 / 27 / 18$ & $67 / 13 / 20$ & 0.07 \\
\hline Echo-structure, Homo-/Heterogeneous, n & $61 / 39$ & $70 / 30$ & $63 / 37$ & 0.38 \\
\hline Calcification, No/Yes, n & $93 / 7$ & $97 / 3$ & $99 / 1$ & 0.07 \\
\hline Necrosis, No/Yes, n & $99 / 1$ & $97 / 3$ & $95 / 5$ & 0.25 \\
\hline No. of needle passes, median (IQR), n & $2(1-3)$ & $2(1-3)$ & $2(1-3)$ & \\
\hline Number of slides, median (IQR), $n$ & $11(9-12)$ & $12(11-15)$ & $10(7-12)$ & $<0.001$ \\
\hline Complications, $\mathrm{n}$ & 0 & 0 & 0 & \\
\hline
\end{tabular}

- Table 2 Diagnostic adequacy of the specimen from the three methods of fine-needle aspiration according to site.

\begin{tabular}{|c|c|c|c|c|}
\hline Diagnostic adequacy & Capillary & Suction & No suction & $P$ value \\
\hline Lymph node, No/Yes, n & $7 / 75$ & $8 / 74$ & $5 / 66$ & 0.84 \\
\hline Pancreas, No/Yes, n & $2 / 16$ & $1 / 17$ & $1 / 28$ & 0.56 \\
\hline Total, No/Yes, n & $9 / 91$ & $9 / 91$ & $6 / 94$ & 0.67 \\
\hline
\end{tabular}

- Table 3 Comparison of cytopathological features between the three methods of fine-needle aspiration.

\begin{tabular}{|c|c|c|c|c|}
\hline & Capillary & Suction & No suction & $P$ value \\
\hline Cellularity, $0 / 1 / 2,{ }^{1} \mathrm{n}$ & $0 / 19 / 81$ & $2 / 14 / 84$ & $2 / 20 / 78$ & 0.494 \\
\hline Blood contamination, $0 / 1 / 2,{ }^{2} \mathrm{n}$ & $28 / 63 / 9$ & $11 / 59 / 30$ & $32 / 52 / 16$ & 0.000 \\
\hline Specimen adequacy, ${ }^{3} 0 / 1, \mathrm{n}$ & 9/91 & 9/91 & $4 / 96$ & 0.665 \\
\hline
\end{tabular}

There was no statistically significant difference regarding cellularity and sensitivity. Previously it was believed that the use of suction increased cellularity and sensitivity: Bhutani et al. reported improved cellularity with suction [15]; Puri et al. found that suction was associated with higher sensitivity and negative predictive value [11]; and Lee et al. also demonstrated better cellularity with the suction method [12].

Our study has certain limitations, including the lack of an onsite cytopathological evaluation and the single-center setting. The strengths of the study are that it was a randomized study with a large sample size. Various EUS characteristics and cytopathological features were compared. Furthermore, this is the first study to compare the three methods of FNA. The cytopathologist was blinded to the FNA method used, and this further strengthens the study design.

In conclusion, the capillary action, suction, and no suction methods of EUS-FNA are similar in terms of diagnostic adequacy of the specimen; however, the suction method has the disadvantages of generating more slides and more blood clots. We strongly recommend that a multicenter study with a large 
numbers of patients is conducted to investigate this subject further.

\section{Competing interests}

None

\section{References}

[1] Vilmann P, Jacobsen GK, Henriksen FW et al. Endoscopic ultrasonography with guided fine needle aspiration biopsy in pancreatic disease. Gastrointest Endosc 1992; 38: $172-173$

[2] Shah JN, Ahmad NA, Beilstein MC et al. Clinical impact of endoscopic ultrasonography on the management of malignancies. Clin Gastroenterol Hepatol 2004; 2: 1069-1073

[3] Varadarajulu S, Fockens P, Hawes RH. Best practices in endoscopic ultrasound-guided fine-needle aspiration. Clin Gastroenterol Hepatol 2012; 10: $697-703$

[4] Sahai AV, Paquin SC, Gariépy G. A prospective comparison of endoscopic ultrasound-guided fine needle aspiration results obtained in the same lesion, with and without the needle stylet. Endoscopy 2010; 42: $900-903$

[5] Rastogi A, Wani S, Gupta N et al. A prospective, single-blind, randomized, controlled trial of EUS-guided FNA with and without a stylet. Gastrointest Endosc 2011; 74: 58-64

[6] Wani S, Gupta N, Gaddam S et al. A comparative study of endoscopic ultrasound guided fine needle aspiration with and without a stylet. Dig Dis Sci 2011; 56: 2409-2414

[7] Wani S, Early D, Kunkel J et al. Diagnostic yield of malignancy during EUS-guided FNA of solid lesions with and without a stylet: a prospec- tive, single blind, randomized, controlled trial. Gastrointest Endosc 2012; 76: $328-335$

[8] Gimeno-García AZ, Paquin SC, Gariépy G et al. Comparison of endoscopic ultrasonography-guided fine-needle aspiration cytology results with and without the stylet in 3364 cases. Dig Endosc 2013; 25: $303-307$

[9] Wallace MB, Kennedy T, Durkalski V et al. Randomized controlled trial of EUS-guided fine needle aspiration techniques for the detection of malignant lymphadenopathy. Gastrointest Endosc 2001; 54: 441 447

[10] Storch IM, Sussman DA, Jorda M et al. Evaluation of fine needle aspiration vs. fine needle capillary sampling on specimen quality and diagnostic accuracy in endoscopic ultrasound-guided biopsy. Acta Cytol 2007; 51: $837-842$

[11] Puri R, Vilmann P, Săftoiu A et al. Randomized controlled trial of endoscopic ultrasound-guided fine-needle sampling with or without suction for better cytological diagnosis. Scand J Gastroenterol 2009; 44: $499-504$

[12] Lee JK, Choi JH, Lee KH et al. A prospective, comparative trial to optimize sampling techniques in EUS-guided FNA of solid pancreatic masses. Gastrointest Endosc 2013; 77: 745 - 751

[13] Martin HE, Ellis EB. Biopsy by needle puncture and aspiration. Ann Surg 1930; 92: 169-181

[14] Attam R, Arain MA, Bloechl S] et al. Wet suction technique (WEST): a novel way to enhance the quality of EUS-FNA aspirate. Results of a prospective, single-blind, randomized, controlled trial using a 22gauge needle for EUS-FNA of solid lesions. Gastrointest Endosc 2015; 81: $1401-1407$

[15] Bhutani MS, Suryaprasad S, Moezzi J et al. Improved technique for performing endoscopic ultrasound guided fine needle aspiration of lymph nodes. Endoscopy 1999; 31: 550 - 553 